\title{
A New Approach to DNA, RNA, and Protein Motifs Templates Visualization and Analysis via Compilation Technique
}

\author{
Hussein K. Al-Khafaji ${ }^{1}$, Zainab M.Jameel ${ }^{2}$ \\ ${ }^{I}$ Al-Rafidain University College \\ ${ }^{2}$ University of Information Technology and Communication
}

\begin{abstract}
Motifs template is the input for many bioinformatics systems such codons finding, transcription, transaction, sequential pattern miner, and bioinformatics databases analysis. The size of motifs arranged from one base up to several Mega bases, therefore, the typing errors increase according to the size of motifs. In addition, when the structures motifs are submitted to bioinformatics systems, the specifications of motifs components are required, i.e. the simple motifs, gaps, and the lower bound and upper bound of each gap. The motifs can be of DNA, RNA, or Protein. In this research, a motif parser and visualization module is designed depending on a proposed a context free grammar, CFG, and colors human recognition system. GFC describes the motif structure to parse the motifs, detect, debug the errors, and analyze the motifs template to its components. Many experiments are accomplished using motifs templates of various sizes arranged from 10 Kbase to 10 Mbase, various numbers of gaps arranged from 15 gaps to 15000 gaps, and different numbers of errors arranged from 100 errors to 1820 errors. The proposed systems, in all these experiments, exhibited linear behavior in parsing phase and visualization phase that indicates its scalability to motifs template sizes.
\end{abstract}

\section{Introduction}

This section contains a review for some terms and principles related to bioinformatics, which are necessary to preceed in this research.

\subsection{Bioinformatics}

It is difficult to give exactly the source of bioinformatics, both as a term and as propriety. The expression was employed as early as 1977 by Dutch theoretical biologist PaulienHogeweg when she depicted her key of research as bioinformatics [1].PaulienHogeweginnovated the expression bioinformatics in 1979 for the study of informatics operations in bioticsystems. It was mainly used since late 1980s in genetics and genomics especially in those fields ofgenomics cover a wide area of DNA series. One can define Bioinformatics as the use of computer technology for the control of information in biology. Bioinformatics is concerned with storing, extracting, organizing, analyzing, interpreting and usinginformation from biological sequences and molecules. It has been primarily motivated by progress in DNA sequencingand mapping methods. Recently the rapid progress in genomic and other molecular researchmethods and developments in information technologies have combined to generate a great amount ofinformation on molecular biology [2]. The main goal of bioinformatics is to enhance the understanding of biological activities. The differentiating factor that distinguishes bioinformatics from other techniques, however, is its concentration on developing and using computationally intensive methods to carry out this goal. Examples cover pattern recognition, data mining, machine learning algorithms, and visualization. Major research attempts in the area cover sequence alignment, gene discovery, genome assembly, drug design, drug discovery, protein structure alignment, protein structure prediction, prediction of gene expression and protein-protein interactions, genome-wide association studies and the evolution modeling [3].

\subsection{Deoxyribonucleic Acid (DNA)}

A double helix molecule is composed of a linear nucleotides structure (See Figure 1). DNA carries the genetic code for an organism in the order of the bases. The double helix of DNA that comes from the hydrogen bonds is formative between bases when two polynucleotide chains are identical, but employment in other directions. The information in DNA is stored as a code consist of chemical bases contain of about three billion bases. The sequence of these bases determines the information available for constructing and maintaining an organism. DNA bases contained four nucleotides (A) adenine, $(\mathrm{T})$ thymine, $(\mathrm{C})$ cytosine, $(\mathrm{G})$ guanine that joined with each other, (A) with (T) and (C) with (G), to make base pairs. Each base is also attached to a sugar molecule and a phosphate molecule. Nucleotide contains a base, sugar, and phosphate. Nucleotides are placed in two long strands that make a double helix. An essential Characteristics of DNA is that it can replicate itself. In the double helix of DNA each strand can be used as a pattern for duplicating the sequence of bases. This is very important because when cells divided each new cell need a delicate copy of DNA that found in the old cell [4]. 


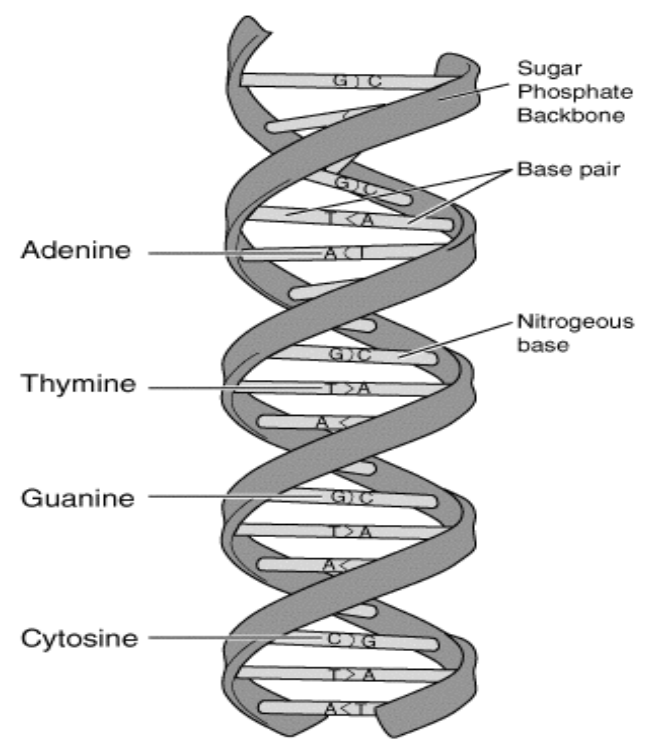

Figure (1) DNA is a double helix formed by base pairs [4]

\subsection{Ribonucleic Acid (RNA)}

Is a sequence of nucleotidessimilar to DNA, RNA nucleotides containing ribose rings in state of deoxyribose .RNA bases contained four nucleotides (A) adenine, (U) uracil , (C) cytosine, (G) guanine that joined with each other, (A) with (U) and (C) with (G), to make base pairs [4]. Figure (2) show the difference between RNA and DNA.The major function of RNA is to carry the genetic code which is important in establishing of proteins from the nucleus to the ribosome. RNA contains multiple types the main types are:

1) (mRNA) messenger RNA: which is mounted from a DNA gene. Messenger ribonucleic acid contains information of the main sequence of amino acids in protein to be mounted. Messenger RNA responsible to move the code into cytoplasm which protein mounted.

2) (tRNA) transfer RNA :which is contain twenty different transfer ribonucleic acid ,one for each of amino acid. The transfer RNA reads the messenger RNA "codon" by utilization its own "anticodon". The actual reading is done during the match pairs through hydrogen bonds.Every "codon" is reading by multiple Transfer RNA until suitable matching of the "anticodon" with the "codon" happen.

3) (r RNA )ribosomal RNA: through the cytoplasm protein and ribosomal RNA joined to each other from a nucleoprotein dub a ribosome the main job of ribosomal RNA that move the enzymes for to create protein[5].

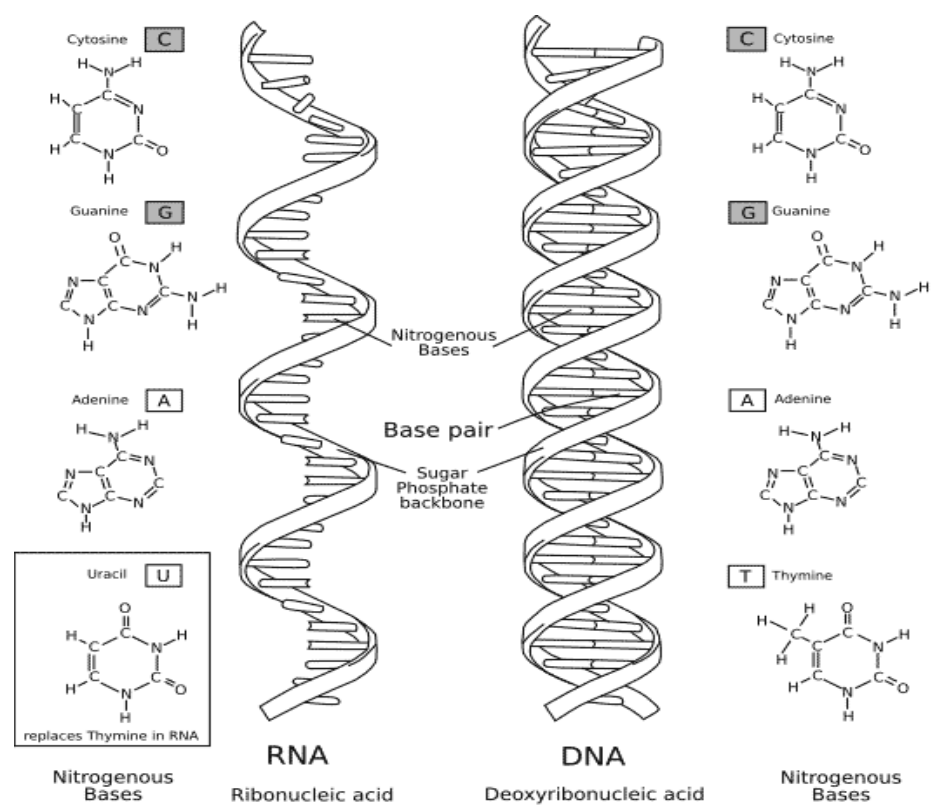

Figure (2) depicted differences between DNA \& RNA [4] 


\subsection{Protein}

A Protein is composed of small unit called amino acids [6]. There are more twenty type of amino acid. Proteins are containing different type of amino acids. Proteins are large molecules contain multiple hundred of amino acids in order of sequence. The main job of protein is build and repairs a body [7]. Proteins contain twenty bases are (A) adenine, (T) thymine, (C) cytosine, (G) guanine, (I) isoleucine, (D) aspartic acid, (E) glutamic acid, $(\mathrm{F})$ phenylalanine, $(\mathrm{K})$ lysine, $(\mathrm{R})$ arginine, $(\mathrm{H})$ histidine, $(\mathrm{N})$ asparagines, $(\mathrm{Q})$ glutamine, $(\mathrm{S})$ serine, (L) leucine, (V) valine, (W) tryptophan, (Y) tyrosine, $(\mathrm{M})$ methionine and $(\mathrm{P})$ proline that order in sequence[8].

\subsection{Motif \&Motif Template}

Motif is used in two ways in the structure. First way indicates sequence of amino acid that is referring to properties of a special biochemical task. Second way, motif represents as a collection of contiguous structure elements that either select a part of task significance or select a part of folded domain independently [9].

Motifs composed of two types simple or structure motif; a simple motif also named single pattern. Simple motif does not contain gaps, that separate simple motif because easy to matching relatively short bases, and the second type is structure motif also named compound pattern, structure motif contains gaps for this reason named structure motif, that approach of it is more difficult than simple motif [3].

DNA motif is a serial of a nucleic acid pattern.DNA motif contacts with structural motifs in proteins. Motifs might happen in each of the strands of DNA [10]. DNA motif contains four bases A, T, C and G. the shape of DNA motif pattern is ATACCGATTTCGCGCGCGTT and sometimes contain gaps such as AATATA $[3,5]$ CTG[2,4] AATGCCG.

RNA motif is defined as a sequence of levels that occurs a short sequences in functional RNAs, for examples (tRNA) transfer RNA or (rRNA) ribosomal RNA [11]. RNA motif contains four bases A, U, C and G. the final form of RNA motifs is AUGUUCGAGGGCAUU and sometimes RNA motifs contain gaps in the form such as AUU[5,8]CCCG[7,9]AAAUGGC.

A protein motif is asequence of amino acid found in proteins. Change the motif according to biological function [12]. Protein motifs contain twenty bases in form that A, T, C, G, I, E, D, F, M, Y, Q, N, H, S, L, V, W, R, K and $\mathrm{P}$. the final form of protein motif is RQWPPAATTTVDE and sometimes contain gaps such as RQWPP[3,6]TAQWVF.

\subsection{Motif Template}

Definition of Motif template contains a sequence of bases and gaps. These contain lower number and upper number. Simple motif consists of bases for any type of motif that used. The general structure of motif represented by the form:

$\mathrm{M}_{1}\left\{\left[1_{1}, \mathrm{u}_{1}\right] \mathrm{M}_{2}\left\{\left[\mathrm{l}_{2}, \mathrm{u}_{2}\right] \mathrm{M}_{3}\right\}\left\{\ldots . . \mathrm{M}_{\mathrm{n}}\left[\mathrm{l}_{\mathrm{n}}, \mathrm{u}_{\mathrm{n}}\right]\right\} \mathrm{M}_{\mathrm{n}+1}\right\}$...

So, for example consider the following protein motif:

KMKKVVVVVQ $[8,10]$ AVCCEW $[2,3] \mathrm{CW}$

It is a structure motif template which consists of three simple motifs in protein alphabets, M1 [8, 10] M2 [2, 3] M3 such that:

- $\quad$ M1 is KMKKVVVVVQ with length of 10 bases.

- $\mathrm{M} 2$ is AVCCEW with length of 6 bases.

- $\mathrm{M} 3$ is CW with length of 2 bases.

- $[8,10]$ is the gap between two simple motifs as lower bound of 8 bases and upper bound of 10 bases.

- $[2,3]$ is the gap between two simple motifs as lower bound of 2 bases and upper bound of 3 bases.

The lower bound must be less than or equal upper bound and integer number.

According to the previous introduction, the reader can conclude that a motif is a sequence of alphabets contains bases and gaps. The motif contains millions of bases in accordance with its uses. This paper produces a new parser to detect and correct the errors in the motif templates before further processing such as sequential pattern mining in bioinformatics system, cordon finding, etc. In addition, the paper introduces a design and implementation of a motifs visualization facility. The next subsections describe the design and implementation of this motif template parser and visualization facility in details.

\section{The Proposed System of Motifs Template Parsing And Visualization}

The following subsection describes the design of the motif template parser and visualization system.

\subsection{The proposed system of Motifs Template parsing}

The general structure of the motif parser is show in figure (3). Motif source selector enables user to input the motif template using the keyboard, (for small size motif), or to load a motif from data set. 


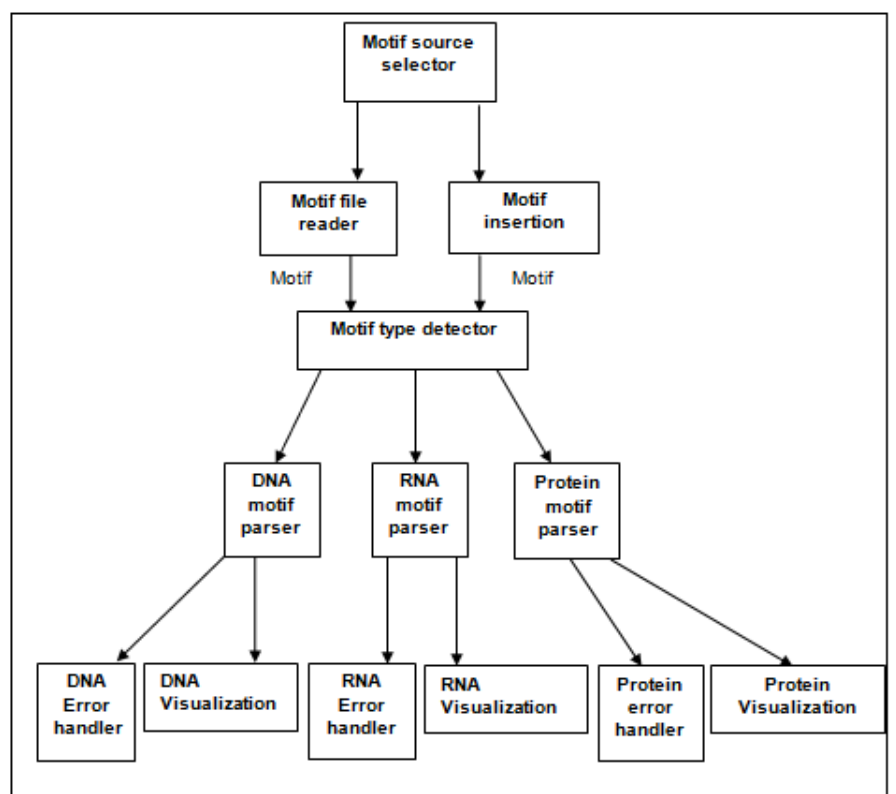

Figure (3) architecture of motif parsing and visualization system

Motif files reader loads and segments huge files according to memory size. Motif type detector is responsible for determining the type of motif template. According to the type of the Motif, suitable parser will treat it. If the Motif template passed the parsing process, specialized visualization module will visualize it.

As Motif has fixed structure and it as well as its components cannot be changed it must always be correct and without errors (both oral and grammatical) because it is used to give accurate results.

In this research, a parser is proposed which is similar to a standard parser to detect the syntax and semantic errors in addition to intended and unintended noise. As shown in Figure (4), It consists of the following phases:-

1) Lexical analyzer

2) Syntax analyzer

3) Semantic analyzer

4) Motifs and gaps generator

Introducing Motif Pattern into the program either directly or through a file already stored in the computer will correct the error by passing it through several stages in the compiler. The result will appear in the form of a report showing whether there is an error or not. The Motif contains bases and gaps and it should be error-free to be ready for mining process. Three types of Motif were processed in this research; they are DNA, RNA and Protein.

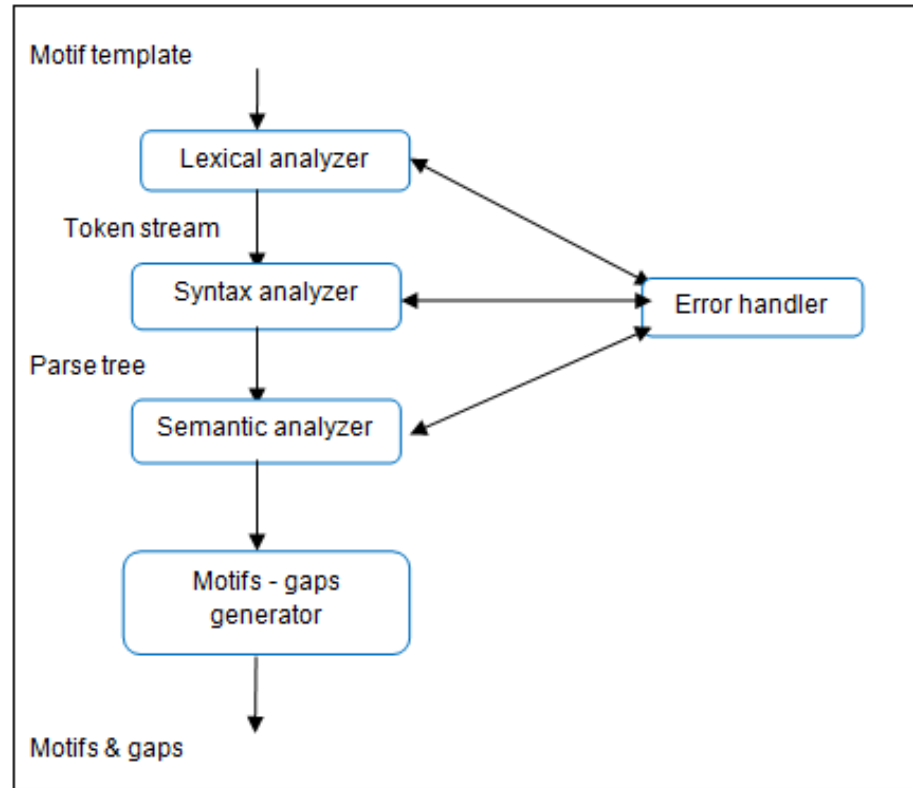

Figure (4) phases of the Motif template parser [3] 


\section{1) Lexical analyzer}

Lexical analysis is the first phase of a parser. It takes the source motif (motif template) from one of the types of resources; keyboard or file. The lexical analyzer decomposes the structure of the Motif template into a number of tokens, by eliminating any whitespace or comments in the source motif. If a token is found invalid by the lexical analyzer, the latter produces an error. The lexical analyzer operates closely with the syntax analyzer. Motif streams are read from the Motif template, legal tokens are checked and the data are passed to the syntax analyzer when it demands. The alphabets of DNA, RNA, and protein are shown in table (1).

Table (1) Alphabets of DNA, RNA, and protein

\begin{tabular}{|l|l|}
\hline Number & $\{0,1,2,3,4,5,6,7,8,9\}$ digit for gap. \\
\hline Strings & $\begin{array}{l}\text { DNA }=\{A, T, C, G\} \\
R N A=\{A, U, C, G\} \\
\text { Protein }=\{l, T, P, G, K, R, C, N, Q, S, L, V, F, W, Y, H, M, A, E, D\}\end{array}$ \\
\hline Special Symbols & Comma(,),Parentheses ([,]) \\
\hline
\end{tabular}

To elucidate the duty of the lexical analyzer, consider the following Motifs, which represent DNA, RNA, and Protein Motifs respectively.

1. ATCT $[1,10] \mathrm{CC}$.

2. AUGC[3,34]GC.

3. ADEFH[23,67]DE.

The result of lexical analyzer phase related to the Motif templates are shown in table (2), table (3), and table (4) respectively.

Table (2) DNA tokens
\begin{tabular}{|l|l|}
\hline $\begin{array}{l}\text { DNA } \\
\text { token } \\
\text { values }\end{array}$ & \multicolumn{1}{|c|}{ Description } \\
\hline ATCT & Simple DNA motif \\
\hline[ & Left side bracket gap \\
\hline 1 & Low integer number \\
\hline, & Comma \\
\hline 10 & High integer number \\
\hline$]$ & right side bracket gap \\
\hline CC & Simple DNA motif \\
\hline
\end{tabular}

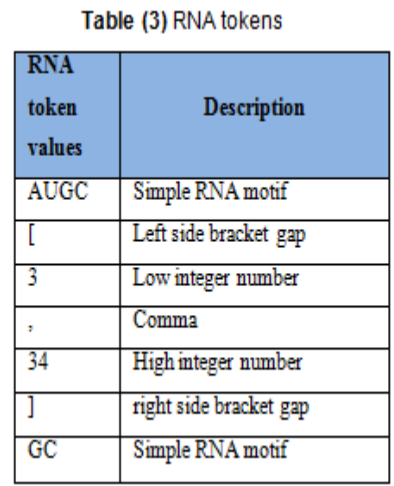

Table (4) protein tokens
\begin{tabular}{|l|l|}
\hline $\begin{array}{l}\text { Protein } \\
\text { token } \\
\text { values }\end{array}$ & description \\
\hline ADEFH & Simple protein motif \\
\hline[ & Left side bracket gap \\
\hline 23 & Low integer number \\
\hline, & Comma \\
\hline 67 & High integer number \\
\hline$]$ & right side bracket gap \\
\hline DE & Simple protein motif \\
\hline
\end{tabular}

Figure (5), figure (6), and figure (7) depict the skeleton of the lexical analyzer. Indeed, they are written in selfdocumentation style, therefore, there is no extended explanation for the steps of the algorithms.

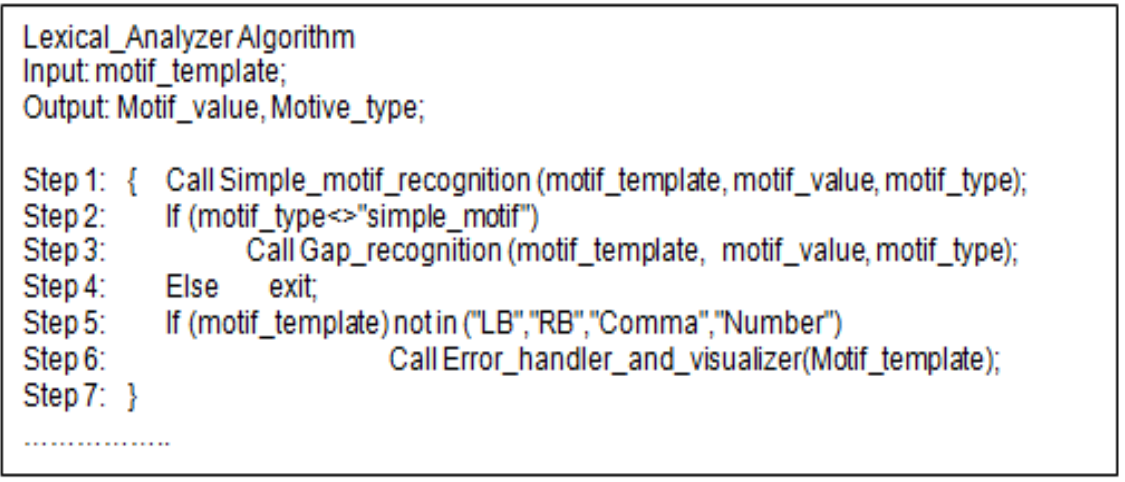

Figure (5) skeleton of lexical analyzer algorithm 


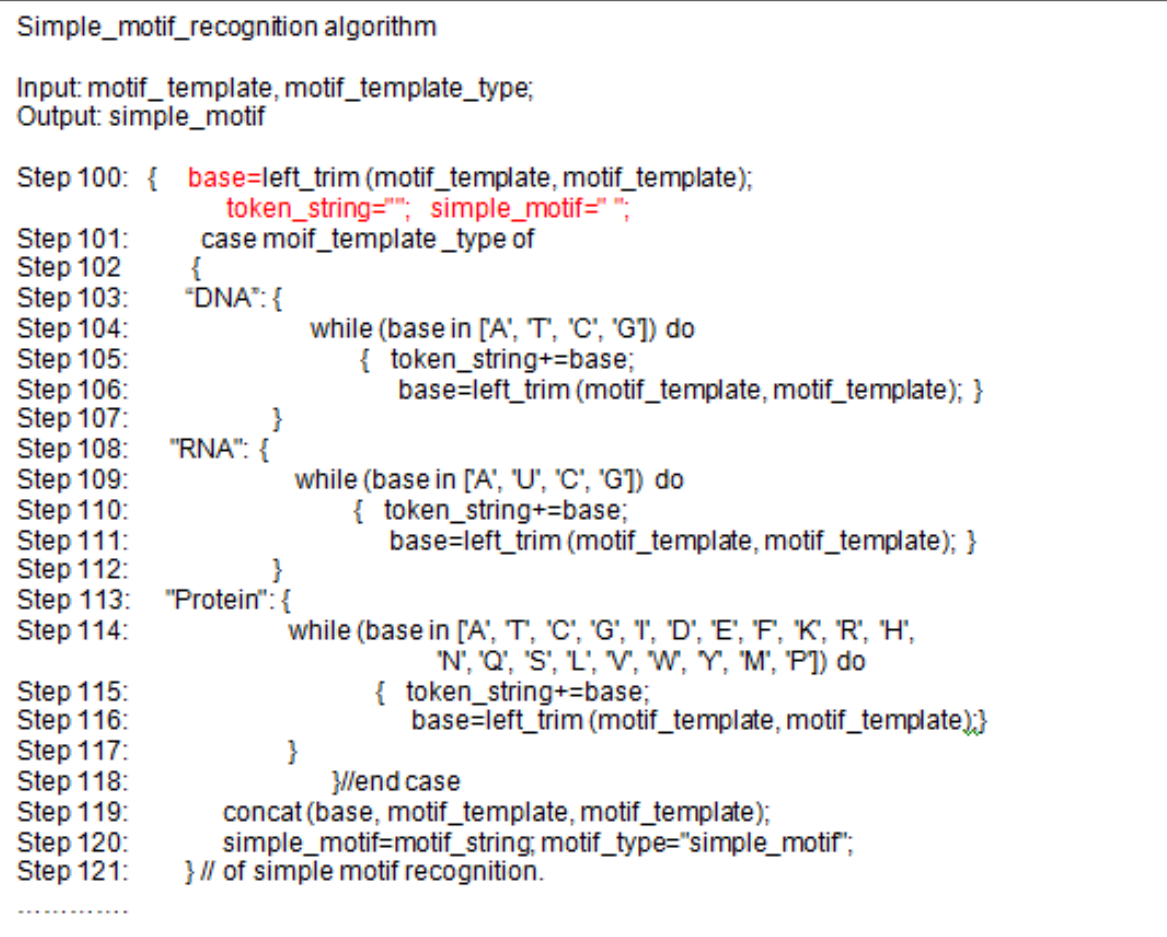

Figure (6) simple motif recognition algorithm

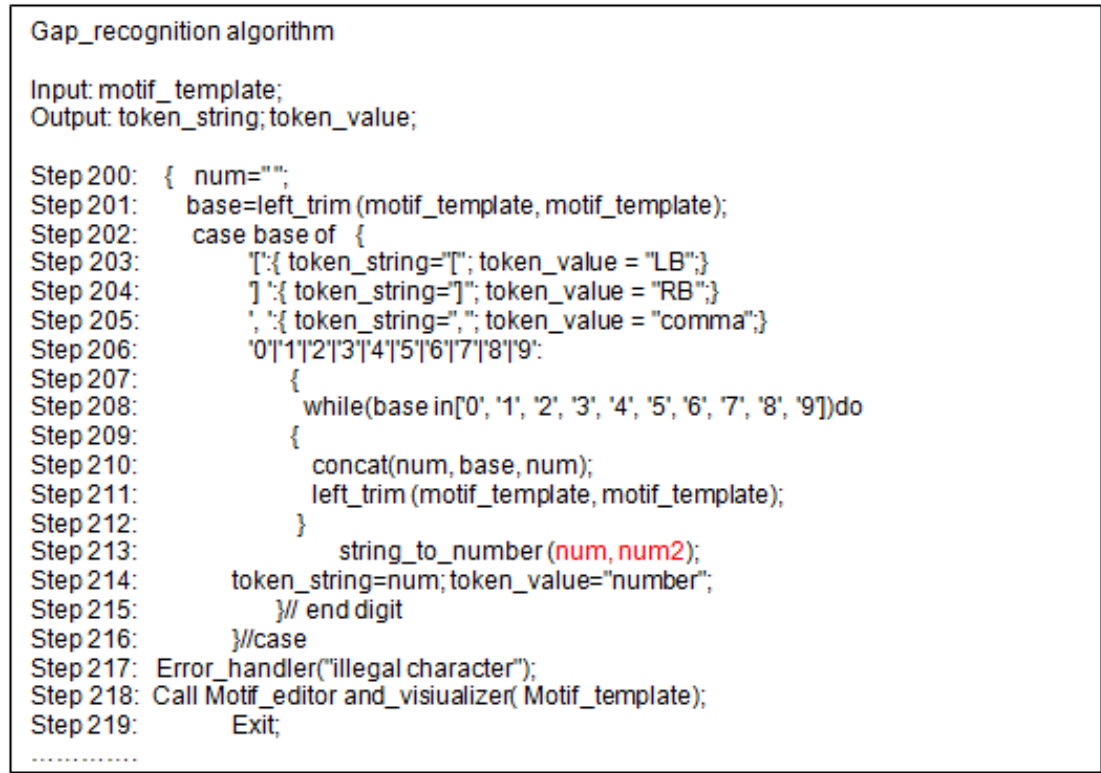

Figure (7) Gap recognition algorithm

\section{2) Syntax Analyzer}

A syntax analyzer, also known as parser, receives the input from a lexical analyzer in the form of token flow. The source motif, (token stream), is analyzed by the parser against the production rules to discover any mistake in the motif template.

The Parser is supposed to parse the entire Motif even though some errors are found in the Motif template. The Parser uses strategies for error recovering called context free grammar (CFG), which is illustrated in figure (8). A parse tree is the output of this phase. The leaves of this tree are the tokens of lexical analyzer phase, and these leaves read from left to right. The parse tree of motif is illustrated in figure (9). In this way the parser performs two functions, i.e., parsing the Motif template, searching for mistakes and produces a parse tree as the output of the phase. 


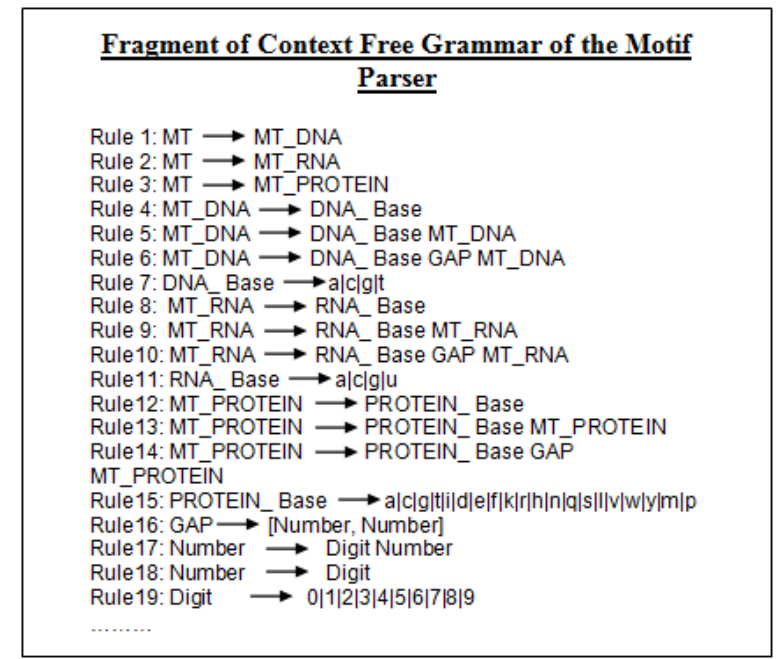

Figure (8) context free grammar rules of motif parser

Accordingly, if the protein motif template, ID $[1,3] \mathrm{N}[2,4] \mathrm{M}$ is correct ,then the validation process will be as follows:

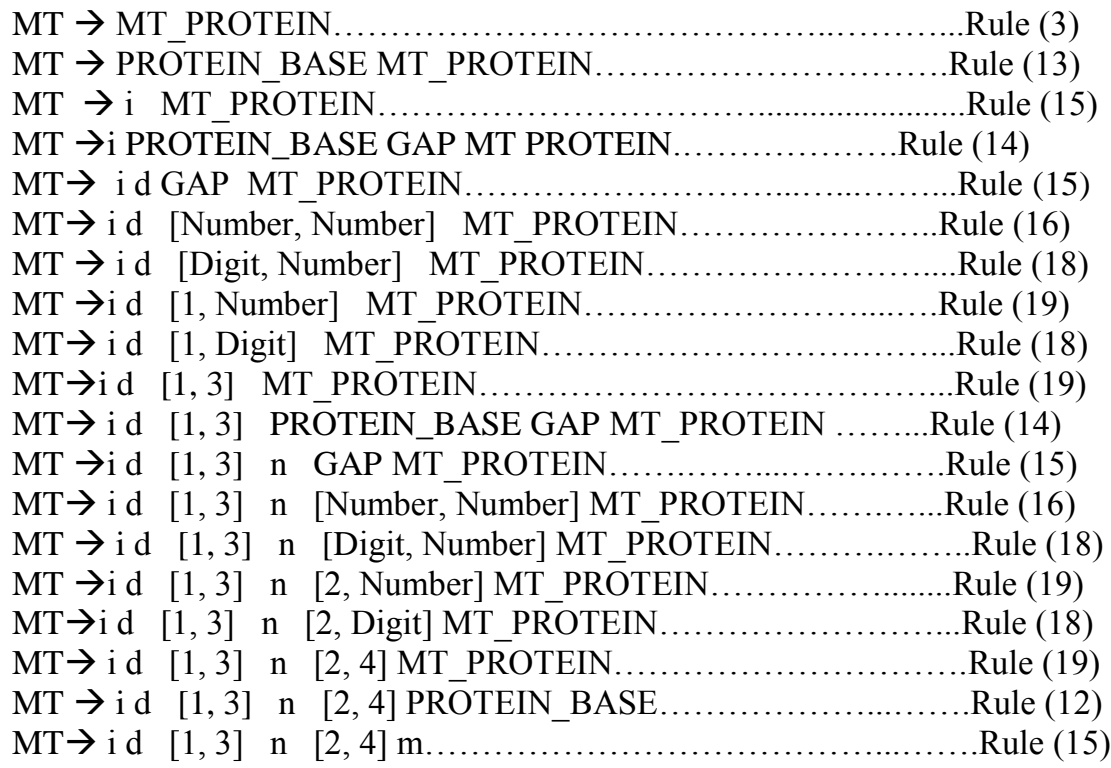

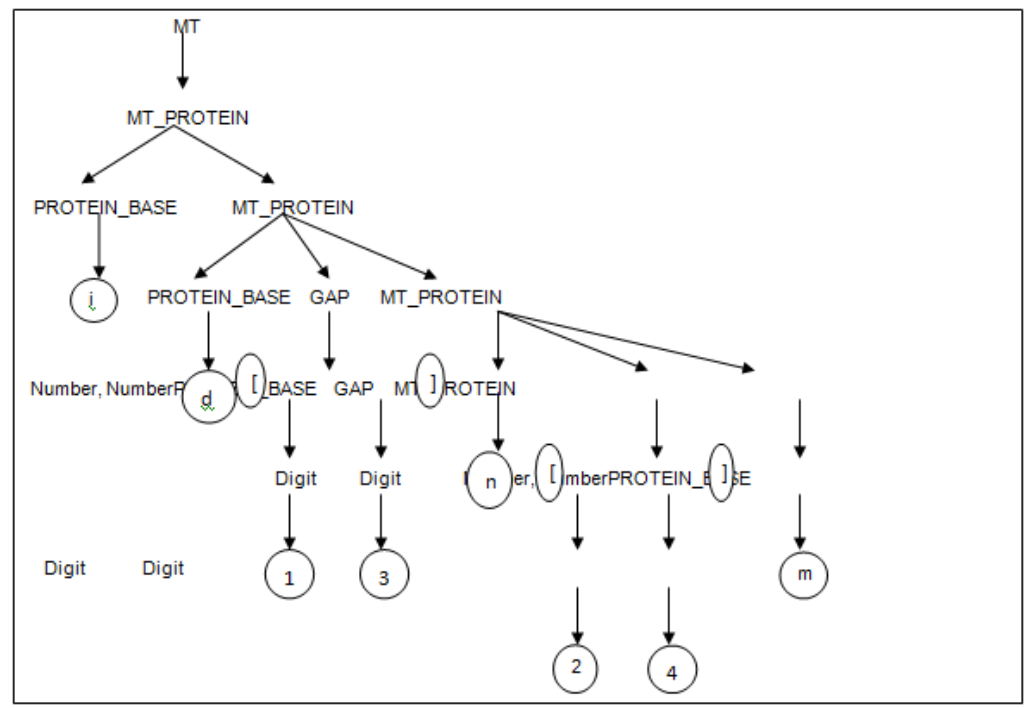

Figure (9) protein motif template parse tree 


\section{3) Semantic Analyzers}

Meaning is provided to Motif constructs by its semantics, like tokens and syntax structure. Semantics help interpret symbols, their kinds, and their relationships with each other. Semantic analysis decides whether the syntactical structure made in the source Motif derives any meaning or not it should not issue an error in lexical and syndical analysis phase, as it is lexically and structurally correct, but it should produce a semantic mistake as the kind of the assignment varies. The grammar of the Motif sets these rules which are evaluated in semantic analysis. The following is some of semantic errors that can be manipulated by the semantic analyzer, figure (10) shows the semantic phase deals with motif in specific rules:

1) The right number in gap in the motif must be valued smaller than left hand side value.

EX: DNA motif $=$ AT $[\underline{5}, \underline{7}]$ CCGA

RNA motif $=$ UUA $[\underline{3}, \underline{6}]$ UACA.

2) Low and high number in gap in the motif must be integer.

EX: Protein motif $=$ KQVV $[2,9]$ RRTC

EX: DNA motif $=$ ATC $[2,2]$ CCGA

3) The gap interval must not exceed the size of data set to be mined.

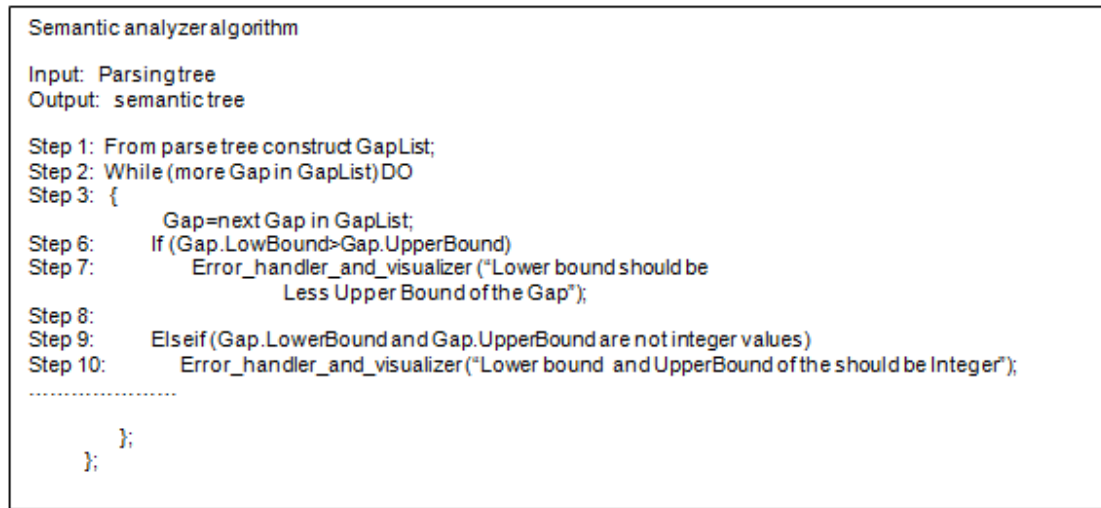

Figure (10) fragment of semantic analyzer algorithm

\section{4) Motifs \& Gaps generator}

Motifs and Gaps generator can be considered as the final phase of the proposed parser. The output of these phase considered in two tables, the first one named simple motifs table contain bases of simple motif, real position of motif contain (start real position, end real position and length of simple motif), and position of motif contain (start position, end position, and length of motif). The second table consists of gaps, lower bound in gap, upper bound in gap, start position of gap and end position of gap; you can see these tables with protein examples in figure (11).

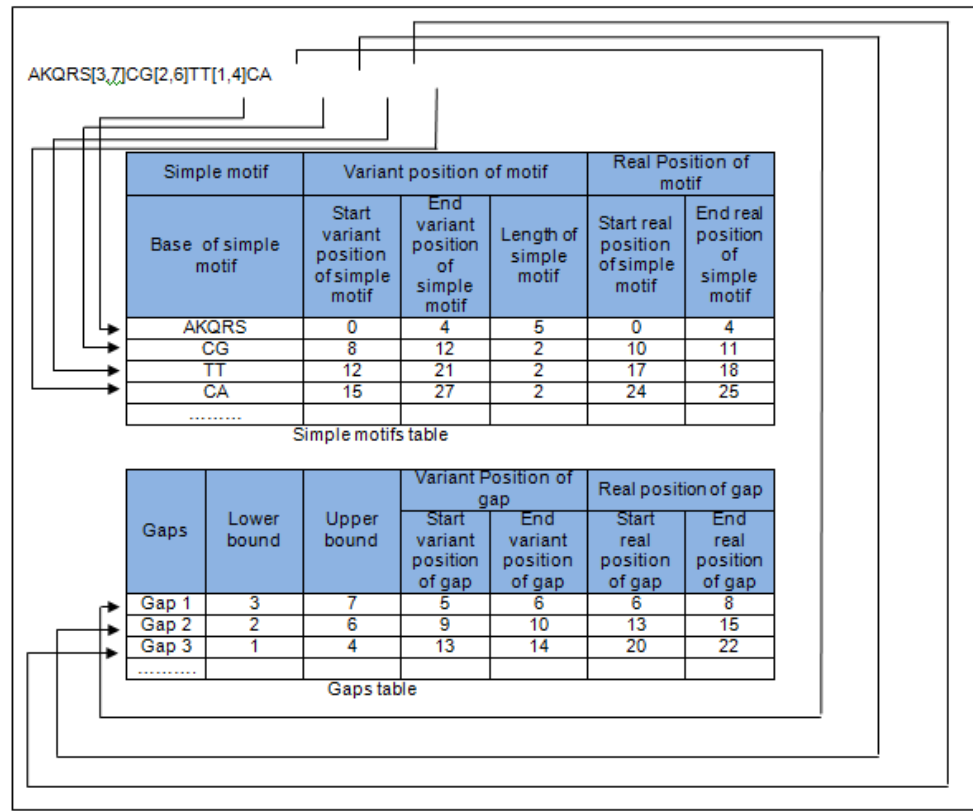

Figure (11) output of Motif and Gaps generator phase 


\subsection{The proposed system of Motifs Template visualization}

A colorized system for the motif can be made by giving a color to every element of the Motif whether it is a symbol, number or characters. Figure (12) illustrates the colors used for this purpose. Tables (5) shows the colors specified for the alphabet of DNA, RNA, protein, and other symbols (legal and illegal). The black color is assigned for illegal symbols.

Colors will activate human colors recognition system and as a result, the time of error detection and correction degenerated in addition to minimization of error percentage.

The visualization module helps out in:

1- Activation of user concentration on errors.

2- Reducing the time consumed in finding the error.

3- Improving the way to reach an error through its pointer.

4- Accuracy in correction of errors.

5- Improving the overall performance of the system.

Table (5) DNA \& RNA \& protein \& other symbols (legal and illegal) colors

\begin{tabular}{|c|c|c|c|}
\hline & Characters \& symbols & Description & Color \\
\hline 1 & A & Adenine & Green \\
\hline 2 & $\mathrm{~T}$ & Thymine & Red \\
\hline 3 & $\mathrm{C}$ & Cytosine & Blue \\
\hline 4 & $\mathrm{G}$ & Guanine & Orange \\
\hline 5 & $\mathrm{U}$ & Uracil & Deep pink \\
\hline 6 & $\mathrm{I}$ & Isoleucine & Tan \\
\hline 7 & $\mathrm{D}$ & Aspartic Acid & Dark Goldenrod \\
\hline 8 & $\mathrm{E}$ & Glutamic Acid & Gold \\
\hline 9 & $\mathrm{~F}$ & Phenylalanine & Olive \\
\hline 10 & $\mathrm{~K}$ & Lysine & Dark violet \\
\hline 11 & $\mathrm{R}$ & Arginine & Maroon \\
\hline 12 & $\mathrm{H}$ & Histidine & Light Coral \\
\hline 13 & $\mathrm{~N}$ & Asparagine & Rosy Brown \\
\hline 14 & $\mathrm{Q}$ & Glutamine & Lime green \\
\hline 15 & $\mathrm{~S}$ & Serine & Royal blue \\
\hline 16 & $\mathrm{~L}$ & Leucine & Mid night blue \\
\hline 17 & $\mathrm{~V}$ & Valine & Dodger blue \\
\hline 18 & $\mathrm{~W}$ & Tryptophan & Light sea green \\
\hline 19 & $\mathrm{Y}$ & Tyrosine & Tomato \\
\hline 20 & $M$ & Methionine & Crimson \\
\hline 21 & $\mathrm{P}$ & Proline & Dark sea green \\
\hline 22 & ] and [ & $\begin{array}{l}\text { right side bracket gaps \& } \\
\text { left side bracket gaps }\end{array}$ & Saddle brown \\
\hline 23 & , & Comma & Lamp \\
\hline 24 & \begin{tabular}{l|l|l|l|l|l|l|l|l}
0 & 1 & 2 & 3 & 4 & 5 & 6 & 7 & 8 \\
\end{tabular} & Digit & Deep sky blue \\
\hline 25 & illegal symbols & $\begin{array}{l}\text { Any symbols is not defined } \\
\text { within the alphabet CFG of } \\
\text { motif }\end{array}$ & Black \\
\hline
\end{tabular}

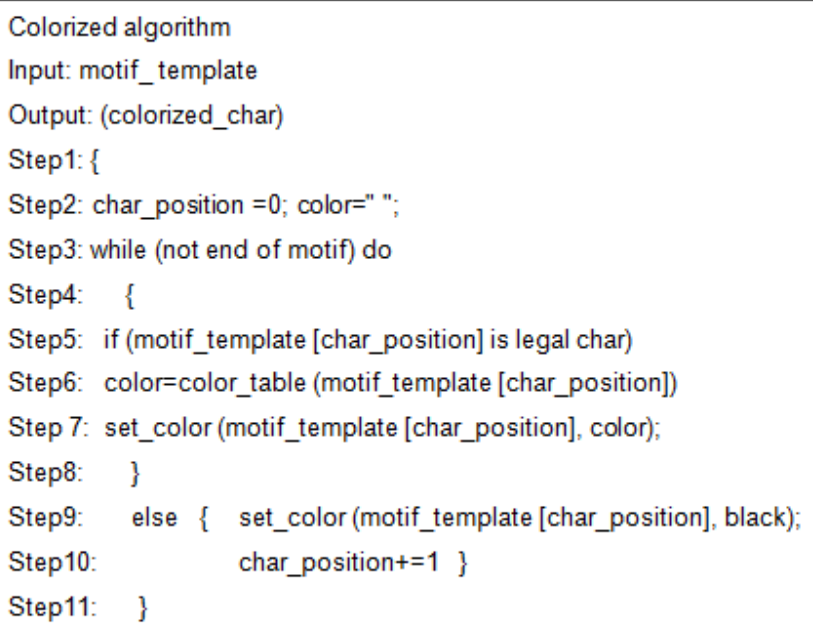

Figure (12) colorizing algorithm 


\section{Experment Results}

This section presents the results of the experiment that focus on the efficiency of the proposed parser to detect and debug the error included in a motif template. The experiments used various motif template sized up to 10000000 bases. The time of processes depends on the size of motif.The proposed parser that proved efficiency to load motif, colorization motif, and handle the errors to huge number of motifs within acceptable time.

Table (6) and figure (13) illustrate the relation between motifs size, number of characters, number of errors, processing time, and time consumed to load and visualizing the entered motif.

Table (6) time of parsing and colorization for various size + error motif

\begin{tabular}{|l|l|l|l|l|l|l|}
\hline Size of motif & $\begin{array}{l}\text { No. of character } \\
\text { in motif }\end{array}$ & $\begin{array}{l}\text { No. of } \\
\text { simple motif }\end{array}$ & No. of gap & $\begin{array}{l}\text { No. of } \\
\text { error }\end{array}$ & $\begin{array}{l}\text { Time of loading } \\
\text { and colorized file }\end{array}$ & $\begin{array}{l}\text { Time of parser } \\
\text { H:M:S:MS }\end{array}$ \\
\hline $10 \mathrm{k}$ base & 10249 & 10172 & 15 & 100 & $0: 0: 0: 742$ & $0: 0: 3: 262$ \\
\hline $50 \mathrm{k}$ base & 51245 & 50860 & 75 & 508 & $0: 0: 2: 738$ & $0: 0: 3: 356$ \\
\hline $100 \mathrm{k}$ base & 102490 & 101720 & 150 & 650 & $0: 0: 5: 012$ & $0: 0: 5: 998$ \\
\hline $500 \mathrm{k}$ base & 512450 & 508600 & 750 & 992 & $0: 0: 17: 578$ & $0: 0: 6: 332$ \\
\hline $1000 \mathrm{k}$ base & 1024900 & 1017200 & 1500 & 1002 & $0: 0: 38: 581$ & $0: 0: 8: 223$ \\
\hline $5000 \mathrm{k}$ base & 5260234 & 5086000 & 7500 & 1350 & $0: 3: 12: 373$ & $0: 0: 25: 221$ \\
\hline $10000 \mathrm{k}$ base & 10520468 & 10222573 & 15000 & 1820 & $0: 6: 2: 123$ & $0: 1: 54: 832$ \\
\hline
\end{tabular}

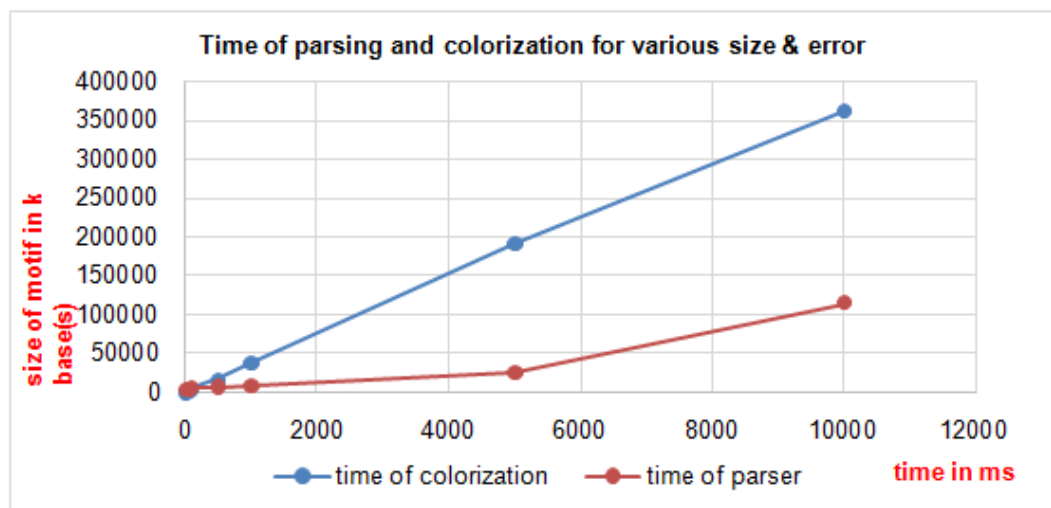

Figure (13) time of parsing and colorization for various size \& error motif

Table (7) and figure (14) illustrate the relation between motifs size, number of characters, processing time, and time consumed to load and visualizing the entered error-free motifs.

Table (7) time of parsing and colorization for various size motif

\begin{tabular}{|l|l|l|l|l|l|}
\hline Size of motif & $\begin{array}{l}\text { No. of character } \\
\text { in motif }\end{array}$ & $\begin{array}{l}\text { No. of } \\
\text { simple motif }\end{array}$ & $\begin{array}{l}\text { No. of } \\
\text { gap }\end{array}$ & $\begin{array}{l}\text { Time of loading } \\
\text { and colorized file }\end{array}$ & $\begin{array}{l}\text { Time of parser } \\
\text { H:M:S:MS }\end{array}$ \\
\hline $10 \mathrm{k}$ base & 10249 & 10172 & 15 & $0: 0: 0: 515$ & $0: 0: 0: 113$ \\
\hline $50 \mathrm{k}$ base & 51245 & 50860 & 75 & $0: 0: 1: 876$ & $0: 0: 0: 082$ \\
\hline $100 \mathrm{k}$ base & 102490 & 101720 & 150 & $0: 0: 3: 646$ & $0: 0: 0: 125$ \\
\hline $500 \mathrm{k}$ base & 512450 & 508600 & 750 & $0: 0: 17: 537$ & $0: 0: 0: 541$ \\
\hline $1000 \mathrm{k}$ base & 1024900 & 1017200 & 1500 & $0: 0: 34: 718$ & $0: 0: 1: 116$ \\
\hline $5000 \mathrm{k}$ base & 5260234 & 5086000 & 7500 & $0: 2: 55: 590$ & $0: 0: 5: 675$ \\
\hline $10000 \mathrm{k}$ base & 10520468 & 10222573 & 15000 & $0: 5: 14: 273$ & $0: 0: 12: 075$ \\
\hline
\end{tabular}

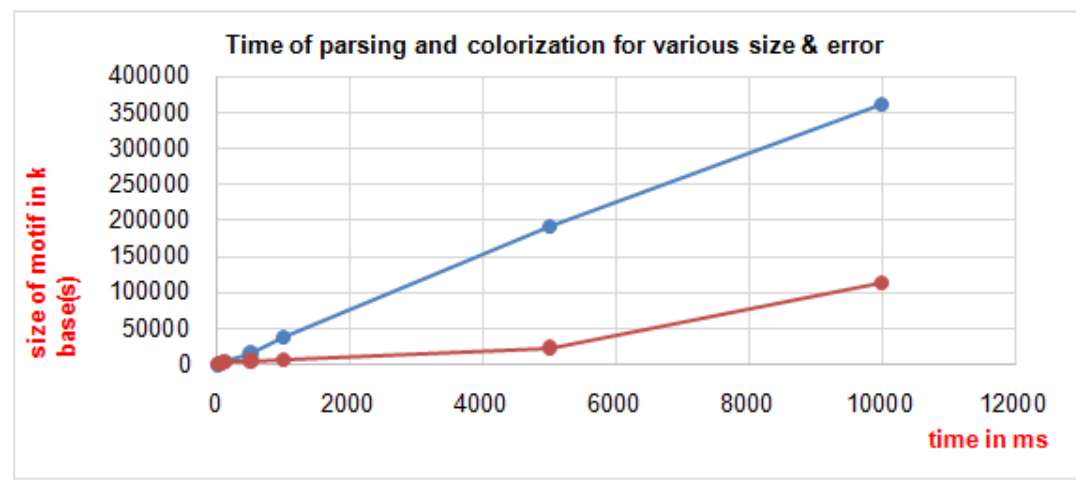

Figure (14) time of parsing and colorization for various size 
Table (8) and figure (15) illustrate the relation between number of error and time of parser for (1000 k bases or $1 \mathrm{M}$ bases), (5000 K bases or $5 \mathrm{M}$ bases) and (10000 K bases or $10 \mathrm{M}$ bases).

Table (8) time of parsing and number of error for size file $1 \mathrm{~m}, 5 \mathrm{~m}$ and $10 \mathrm{~m}$

\begin{tabular}{|l|l|l|l|}
\hline \multirow{2}{*}{ No. of error } & Time of parser & H:M:S:MS & \\
\cline { 2 - 4 } & 1 M bases & 5 M bases & 10 M bases \\
\hline 100 & $0: 0: 1: 051$ & $0: 0: 5: 147$ & $0: 0: 12: 320$ \\
\hline 250 & $0: 0: 1: 092$ & $0: 0: 5: 356$ & $0: 0: 12: 550$ \\
\hline 500 & $0: 0: 1: 110$ & $0: 0: 5: 398$ & $0: 0: 12: 904$ \\
\hline 750 & $0: 0: 1: 147$ & $0: 0: 5: 642$ & $0: 0: 13: 183$ \\
\hline 1000 & $0: 0: 1: 721$ & $0: 0: 5: 685$ & $0: 0: 13: 545$ \\
\hline 1250 & & $0: 0: 6: 001$ & $0: 0: 13: 822$ \\
\hline 1500 & & $0: 0: 6: 626$ & $0: 0: 14: 330$ \\
\hline 1750 & & $0: 0: 6: 904$ & $0: 0: 15: 206$ \\
\hline 2000 & & $0: 0: 7: 034$ & $0: 0: 15: 899$ \\
\hline
\end{tabular}

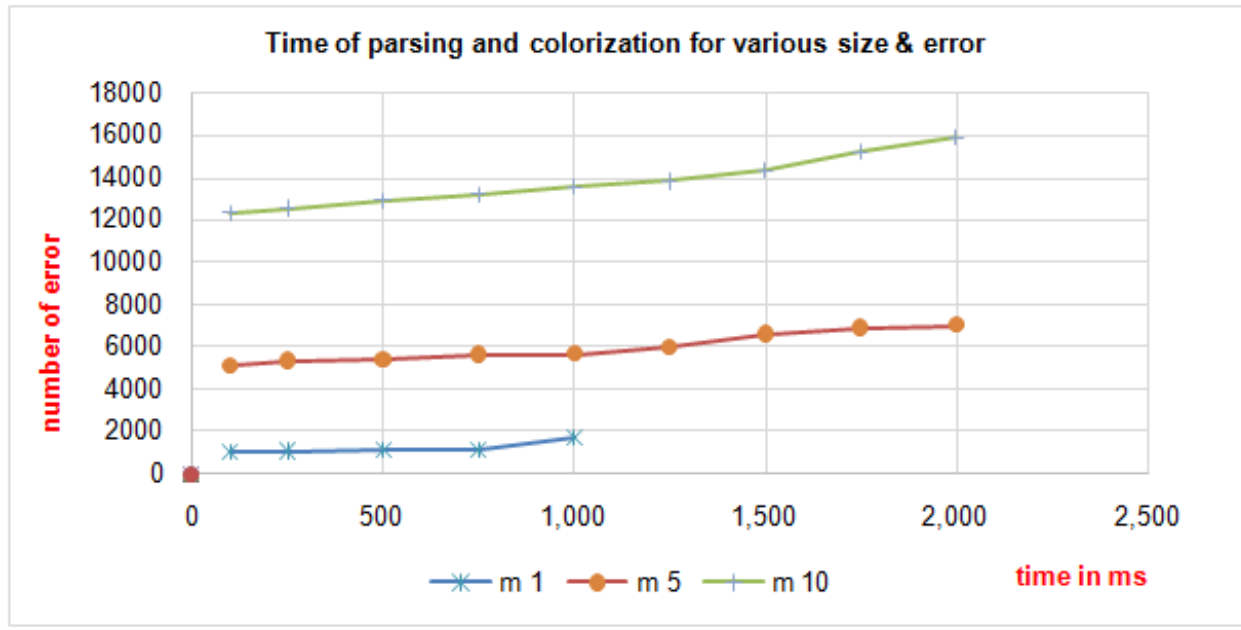

Figure (15) time of parsing and number of error for size file $1 \mathrm{~m}, 5 \mathrm{~m}$ and $10 \mathrm{~m}$

\section{Conclusion}

Many conclusions can be extracted from this research, such as:

1) The compilation technique is very suitable for detection and debugging of the errors and intended and unintended noise in a motif template or bioinformatics databases.

2) The experiments accomplished on the presented system showed its ability to deal with the three types of motif DNA, RNA, and protein efficiently.

3) The proposed system manipulates huge size motifs or bioinformatics databases with various number of errors

\section{Bibliography}

[1]. Attwood T. K., Gisel A., Eriksson N-E., and BongcamRudloff E. "Concepts, Historical Milestones and the Central Place of Bioinformatics in Modern Biology: A European Perspective", Bioinformatics-Trends and Methodologies, 2012.

[2]. PhD. Alla 1 lapidus, bioinformatics and its applications, SPBAU, SPBSU, ST. Petersburg.

[3]. Dr. Hussein K. Al-Khafaji, a New Approach to Motif Templates Analysis via Compilation Technique, Al-Rafidain University Collage, 2014

[4]. SeppHochreiter, bioinformatics I sequence analysis and phylogenetics, institute of bioinformatics, Johannes kepler university linz , winter semester 2013/2014.

[5]. J. Mol. Biol., "Crystal Structure of Yeast Phenylalanine Transfer RNA I. Crystallographic Refinement", 123 607, 1978

[6]. Assistant Professor Sabu M. Thampi, Bioinformatics, LBS College of Engineering.

[7]. Georgia C. Lauritzen, Food \& Nutrition Specialist, What Is Protein, Utah state university, cooperative extension, extension.usu.edu, 1992.

[8]. RashidaHasan\&JainalUddin,Data Mining Techniques for Informative Motif Discovery,International Journal of Computer Applications,February 2014.

[9]. Gregory A Petsko\& Dagmar Ringe, "Protein structure and function", New science press 1td, 2004.

[10]. Modan K Das and Ho-Kwok Dai, "A Survey of DNA Motif Finding Algorithms", Fourth Annual MCBIOS Conference, Computational Frontiers in Biomedicine, 2007.

[11]. Donna K. Hendrix1 , Steven E. Brenner1,2 and Stephen R. Holbrook, RNA structural motifs: building blocks of a modular biomolecule, Cambridge University Press, July 2006.

[12]. Vicki Doronina, How to identify protein motifs from protein sequences, October 2013 